\title{
Deducción de la ecuación de la densidad de corriente en un semiconductor homogéneo no degenerado, en función del tiempo de relajación $\boldsymbol{\tau}_{\boldsymbol{e}}$ para los electrones
}

\author{
Daniel Gonzalo Arboleda Avilés \\ Universidad Central del Ecuador \\ dgarboleda@uce.edu.ec
}

Recibido: 01 de septiembre / Aprobado: 30 de noviembre 2017

\section{Resumen}

Cuando se estudian los coeficientes cinéticos de los semiconductores, se observa que, en la mayoría de la bibliografía especializada en el tema, no existe un modelo matemático que represente a la densidad de corriente en función del tiempo de relajación para los electrones. Se han publicado varios papers, con datos obtenidos experimentalmente, pero no existe un desarrollo teórico al respecto.

Palabras clave: espacio de fases, densidad de corriente, función de distribución de desequilibrio, tiempo de relajación para los electrones.

\section{Abstract}

When the kinetic coefficients of semiconductors are studied, it is observed that there is not a mathematical model that represents the current density based on relaxation time for electrons. There are several published papers, with data obtained experimentally, but there is no theoretical development in this regard.

Keywords: phase space, current density, distribution function imbalance, relaxation time for electrons. 



\section{Introducción}

L

a historia de los semiconductores se remonta a casi dos siglos atrás. Pero es a partir de 1948, fecha de aparición del transistor, cuando se inicia un acelerado desarrollo de la investigación de las propiedades físicas de los sólidos en general y de los semiconductores en particular.

Tal vez fue Faraday quien en 1833 hiciera la primera observación de importancia de lo que más tarde se conocería como semiconductores.

"Faraday observó que la resistividad eléctrica del sulfato de plata disminuye con el aumento de la temperatura" [1].

Este comportamiento es una de las principales características que diferencian a los semiconductores de los metales.

El estudio de los semiconductores ha llevado al descubrimiento de sus propiedades útiles y a una gran cantidad de aplicaciones prácticas. Este campo cambia tan rápidamente que lo que se diga hoy puede ser incorrecto mañana. Está claro que la investigación acerca de estos materiales nos podrá llevar a cosas nuevas e interesantes.

\section{Densidad de corriente de los semiconductores}

El presente trabajo se iniciará con la determinación de la densidad de corriente de un semiconductor homogéneo - no degenerado.

"Homogéneo porque contiene átomos donantes como aceptores. [2] [9]

No degenerado porque el nivel reducido de Fermi es menor que -1":

$[3]$.

$$
\eta=\frac{F-E c}{K_{B} * T}<-1
$$

$\eta \equiv$ Nivel reducido de Fermi

$F \equiv$ Energía de Fermi

Ec $\equiv$ Energía en la banda

de conducción

$K_{B} \equiv$ Constante de Boltzman

$T \equiv$ Temperatura absoluta 


\section{Superficies isoenergéticas:}

Los portadores de carga eléctrica en los metales y semiconductores son las cuasi partículas: los electrones en las bandas de conducción y los huecos en la banda de valencia. Por sus propiedades estas cuasi partículas difieren considerablemente de las partículas libres. Las propiedades dinámicas de las cuasi partículas vienen determinadas por la dependencia de su energía respecto de los vectores de onda (cuasi impulsos). Esta dependencia se conoce con el nombre de ley de dispersión.

Para todas las partículas libres la Ley de dispersión es estándar:

$$
E(\vec{k})=\frac{\hbar^{2} * k^{2}}{2 m}
$$

Siendo m la masa de la partícula. A diferencia de ésta, la energía de la cuasi-partícula

$E \alpha_{s_{z}}(k)$ en el cristal, es una función periódica compuesta de un vector de onda.

Donde:

$\vec{k} \equiv$ vector de onda reducido

$S_{z} \equiv$ estado de espín

$\alpha \equiv$ resto de números cuánticos

que caracterizan el estado

monoelectrónico

En el espacio de los vectores de onda, la dependencia $E \alpha_{S_{Z}}(\vec{k})$ puede representarse por medio de imágenes geométricas llamadas superncies Isoenergéticas que unen los puntos del espacio $\vec{k}$ a las cuales corresponde una misma energía.

Para una partícula libre las superficies isoenergéticas tienen siempre forma esférica.

Las superficies isoenergéticas de los electrones de la banda de conducción y de los huecos de la banda de valencia tienen forma muy compleja.

El cálculo teórico de estas superficies solo puede hacerse utilizando aproximaciones rústicas, incluso en la red cúbica simple de las superficies isoenergéticas solo son esferas en las proximidades del centro de la zona de Brillouin y en las proximidades de sus ocho vértices.

En las proximidades de los valores extremos $E \alpha_{S_{z}}(\vec{k})$ de dentro de la zona de Brillouin, es decir de los valores $k_{0}$, donde $E \alpha_{s_{z}}\left(k_{0}\right)$ alcanza el valor mínimo o 
máximo, las superficies isoenergéticas en el espacio $\vec{k}$ son cerradas. En las inmediaciones de los puntos $k_{0}$ estas son elipsoides". [4] (Davidov, 1981)

Supongamos que el semiconductor se encuentra en un campo eléctrico homogéneo constante cuya intensidad es $\varepsilon$ : si se aplica al cristal un campo exterior (eléctrico, magnético, térmico) el movimiento de los portadores de carga en el cristal adquiere un carácter dirigido coordinado. Ahora la función de distribución es una función de desequilibrio, que depende de la energía de las partículas, de sus coordenadas y del tiempo:

\section{$f(\vec{r}, \vec{k}, t) \equiv$ función de distribución de desequilibrio.}

Definiendo la concentración de electrones, es decir el número de electrones por unidad de volumen:

$\left[\frac{n}{c m^{3}}\right]$, en estado de desequilibrio se procede así:

- En el espacio de fase del cristal de volumen unitario, separamos el elemento de volumen.

- "El espacio de fase se define como un espacio cartesiano $2 \mathrm{n}$ - dimensional, formado por las coordenadas: $q_{1}, \ldots, q_{n} ; p_{1}, \ldots, p_{n}$ ” [5] (Goldstein, 1979)

- Este se determina por el volumen de espacio ordinario:

$$
d \tau_{r}=d x d y d z
$$

- Y por el volumen del espacio de los cuasi-impulsos:

$$
d \tau_{p}=\hbar^{3} d \tau_{k}
$$

Por lo tanto:

$$
d \Gamma \triangleq d \tau_{r} * d \tau_{p}=d \tau_{r} \hbar^{3} * d \tau_{k}=\hbar^{3} d \tau_{r} * d \tau_{k}
$$

En el elemento de volumen del espacio de fase existen $d \Gamma / \hbar^{3}$ células de fase, en cada una de las cuales pueden existir dos electrones con espines en direcciones opuestas.

Entonces, en el elemento de volumen del espacio de fases, existen:

$2 * \frac{d \Gamma}{h^{3}}$ Estados cuánticos.

Si $f(\vec{r}, \vec{k}, t)$ es la probabilidad de que el electrón se encuentre en estos estados, el número de electrones en el elemento del espacio de fase en el instante " $t$ ” será:

$$
n \equiv \text { probabilidad }
$$


Entonces:

$$
d n=f(\vec{r}, \vec{k}, t) * 2 * \frac{d \Gamma}{h^{3}}(\mathbf{2})
$$

(1) en (2) y además: $\hbar=\frac{h}{2 \pi}$ :

$$
d n=f(\vec{r}, \vec{k}, t) * \frac{d \tau_{k}}{4 \pi^{3}} * d \tau_{r}
$$

Integrando respecto del volumen de espacio ordinario: $d \tau_{r}$ :

$$
d n=f(\vec{k}) * \frac{d \tau_{k}}{4 \pi^{3}}
$$

Estos electrones que se mueven por acción del campo eléctrico a la velocidad crean la densidad elemental de corriente:

$$
\begin{aligned}
d \vec{\jmath} & \propto d n * \vec{V} \\
d \vec{\jmath} & =-e * d n * \vec{V} \mathbf{5})
\end{aligned}
$$

(4) en (5):

$$
d \vec{\jmath}=-e * f(\vec{k}) * \frac{d \tau_{k}}{4 \pi^{3}} * \nabla
$$

Debido a que en el semiconductor existen dos tipos de carga, la densidad total de corriente está dada por:

$$
\begin{aligned}
& \vec{J}=\overrightarrow{J_{n}}+\overrightarrow{J_{p}} \text {, donde: }\left\{\begin{array}{r}
\overrightarrow{J_{n}} \equiv \text { densidad de corriente } \\
\text { para los electrones } \\
J_{p} \equiv \text { densidad de corriente } \\
\text { para los huecos }
\end{array}\right. \\
& \Rightarrow d \vec{\jmath}=d \overrightarrow{\jmath_{n}}+d \overrightarrow{J_{p}} \text { (7) } \\
& \text { (6) en (7): } \\
& d \vec{\jmath}=-e * f_{n(\vec{k})} * \frac{d \tau_{k}}{4 \pi^{3}} * \overrightarrow{V_{n}}+e * f_{n(\vec{k})} * \frac{d \tau_{k^{\prime}}}{4 \pi^{3}} * \nabla_{p}
\end{aligned}
$$

Observación: por convención la carga para los huecos será (+) y la carga para los electrones será (-).

$$
\text { Integrando: } \quad J=-\frac{e}{4 \pi^{3}} \int_{\left(V_{B}\right)} f_{n(\vec{k})} * \nabla_{n} d \tau_{k}+\frac{e}{4 \pi^{3}} \int_{\left(V_{B}\right)} f_{p_{\left(\overrightarrow{k^{\prime}}\right)}} * \nabla_{p} d \tau_{k^{\prime}}
$$


Donde:

$\nabla_{n} \equiv$ velocidad de los electrones

$f_{n} \equiv$ función de distribución

de desequilibrio para los electrones

$\nabla_{p} \equiv$ velocidad de los huecos

$f_{p} \equiv$ función de distribución

de desequilibrio para los huecos

Observación: se debe integrar por toda la zona de Brillouin, porque se trata de una red cúbica y es suficiente examinar la variación entre los límites:

$$
\left[-\frac{\pi \hbar}{a}, \frac{\pi \hbar}{a}\right]
$$

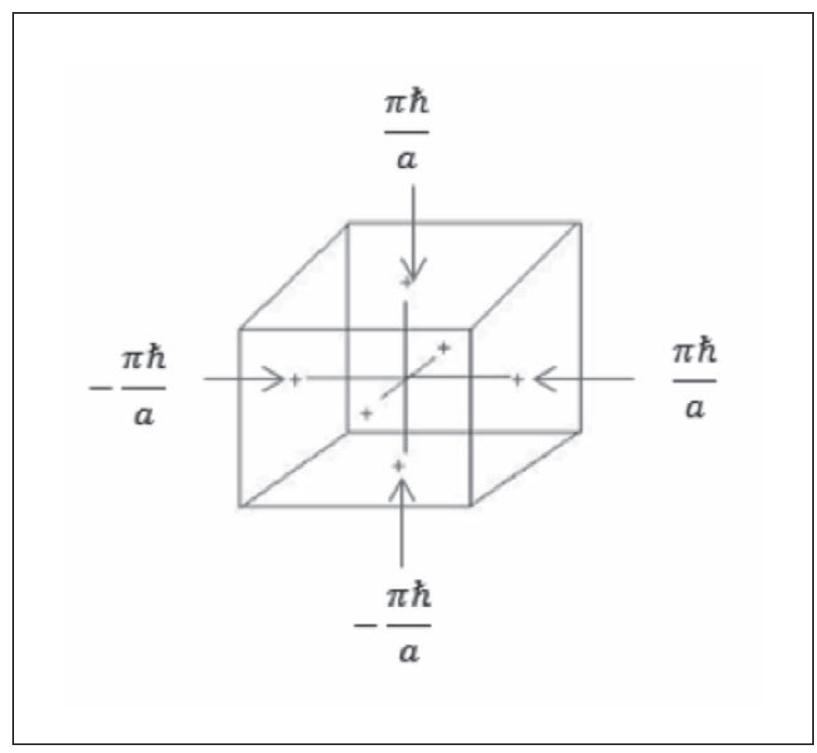

Figura 1. Red cúbica.

Donde: $a \equiv$ constante de la red. [6] (Ashcroft \& Mermin, 1976) 


\section{Materiales y métodos}

\section{Función de distribución de desequilibrio}

La función de distribución de desequilibrio, está dada por la función de distribución de equilibrio más una pequeña perturbación; es decir:

$$
f(\vec{k})=f_{0}(\vec{k})+f_{1}(\vec{k})(\mathbf{9})
$$

Donde:

$$
f_{0}(\vec{k}) \equiv \text { función de distribución }
$$

en equilibrio

$$
f_{1}(\vec{k}) \equiv \text { perturbación }
$$

(9) en (8):

$$
\vec{J}=-\frac{e}{4 \pi^{3}} \int_{\left(V_{B}\right)} \nabla_{n}\left[f_{0}^{(n)}(\vec{k})+f_{1}^{(n)}(\vec{k})\right] d \tau_{k}+\frac{e}{4 \pi^{3}} \int_{\left(V_{B}\right)} \nabla_{p}\left[f_{0}^{(p)}\left(\overrightarrow{\left.k^{\prime}\right)}\right)+f_{1}^{(p)}\left(\overrightarrow{\left.k^{\prime}\right)}\right] d \tau_{k^{\prime}}\right.
$$

Aplicando la propiedad distributiva para el producto y debido a que $f_{0}$ se encuentra en equilibrio termodinámico, entonces $\jmath=0$, para la integral que contiene $f_{0}$ y simplificando, se obtiene:

\section{Ecuación cinética en la aproximación $\tau$}

Planteemos la ecuación cinética en la aproximaciór. $\tau$, la cual proviene de:

$$
\begin{aligned}
& f_{(\vec{k})}=f_{0(\vec{k})}+f_{1(\vec{k})} \therefore f_{1(\vec{k})}=C * e^{-t / \tau} \\
& f_{1(\vec{k})} \equiv \text { perturbación }
\end{aligned}
$$

Esta perturbación puede ser escrita en forma exponencial. 
Entonces:

$$
\begin{aligned}
& f_{(\vec{k})}=f_{0(\vec{k})}+C * e^{-t / \tau} \\
& \frac{f-f_{0}}{C}=e^{-t / \tau} \\
& \ln \left(\frac{f-f_{0}}{C}\right)=-\frac{t}{\tau} \\
& \ln \left(f-f_{0}\right)=-\frac{t}{\tau}+\ln C
\end{aligned}
$$

Diferenciando respecto a $f$ en el miembro izquierdo y respecto a t en el derecho:

$$
\begin{aligned}
& \frac{1}{f-f_{0}} * d f=-\frac{1}{\tau} * d t \\
& \frac{\partial f}{f-f_{0}}=-\frac{\partial t}{\tau} \\
& \left.\frac{\partial f}{\partial t}=-\frac{f-f_{0}}{\tau}\right\} \equiv \text { ecuación cinética }
\end{aligned}
$$

en la aproximación $\tau$ (11)

\section{Resultados}

Deducción de la ecuación de la densidad de corriente en función del tiempo de relajación para los electrones

"Debido a que la función de distribución de desequilibrio está cambiando, por causa de colisiones, es conveniente plantear la ecuación cinética en la aproximación , tonde $1 / \tau$; la tasa de colisiones de los electrones con los fonones".

[6] (Ashcroft \& Mermin, 1976)

(9) en (11) y simplificando:

$\frac{\partial f}{\partial t}=-\frac{f 1_{(\vec{k})}}{\tau}$

Ahora, está por determinarse $f 1_{(\vec{k})}$ : 
Si sabemos que $f=f_{(\vec{r}, \vec{k}, t)}$ y además, se ha dicho que el semiconductor es homogéneo, es decir: la función de distribución de equilibrio más la perturbación $f_{1}$ permanecen constantes con respecto a las coordenadas.

Por lo tanto:

$\nabla_{r} f=0(13)$

También, se sabe que el electrón de energía:

$E=\frac{\hbar^{2} k^{2}}{2 m^{*}}$ (14) $[8]$ (Pankove, 1971)

Donde $\boldsymbol{m}^{*} \equiv$ masa efectiva, la cual puede ser diferente del valor de la masa del electrón en el vacío. El electrón se encuentra inmerso en un campo eléctrico exterior, sobre el cual actúa la fuerza:

$F=\hbar * \frac{d \vec{k}}{d t}=-e * \mathcal{E}(\mathbf{1 5})$

La ecuación (15) proviene de la ecuación semi-clásica del movimiento:

$$
\begin{gathered}
\vec{p}=\hbar * \vec{k} \\
\frac{d}{d t} \vec{p}=\hbar * \frac{d}{d t} \vec{k} \\
F=\hbar * \frac{d}{d t} \vec{k}
\end{gathered}
$$

Puesto que: $f=f_{(\vec{r}, \vec{k}, t)}$

donde $E=E_{k}$

y $\nabla_{r} f=0$,

Entonces $f$ varía solamente con: la energía y el tiempo: $\left.f=f_{(\vec{k}}+1\right)$

Por lo tanto, la variación de la función de distribución, bajo la influencia del campo, se determinará por:

$\left(\frac{\partial f}{\partial t}\right)_{C A M}=\frac{\partial f_{n}}{\partial E} * \frac{\partial E}{\partial \vec{k}} * \frac{\partial \vec{k}}{\partial t}$

Observación: el subíndice n, indica que dicha variación es para los electrones. 
De (15):

$\frac{d \vec{k}}{d t}=-\frac{e \vec{\varepsilon}}{\hbar}$

De (14):

$$
\begin{gathered}
E=\frac{\hbar^{2} k^{2}}{2 m^{*}} \\
\frac{\partial E}{\partial k}=\frac{\hbar^{2}}{2 m^{*}} * 2 k \\
\frac{\partial E}{\partial \vec{k}}=\frac{\hbar^{2}}{m^{*}} * \vec{k}
\end{gathered}
$$

Sustituyendo (17) y (18) en (16):

$$
\left(\frac{\partial f}{\partial t}\right)_{C A M}=-\frac{e \hbar}{m^{*}} * \frac{\partial f_{n}}{\partial t} *(\vec{k} \cdot \varepsilon) \text { (19) }
$$

Para obtener $f_{1} \stackrel{(n)}{(k)}$ reemplazamos la ecuación (19) en (12):

$f_{1} \stackrel{(n)}{(\vec{k})}=\frac{\tau_{(\vec{k})} e \hbar}{m_{n}^{*}} * \frac{\partial f_{n}}{\partial t} *(\vec{k} \cdot \varepsilon)$

Sustituyendo la ecuación (20) en (10), para el caso de los electrones:

$\vec{J}_{n}=-\frac{e}{4 \pi^{3}} * \int_{\left(V_{B}\right)} \nabla_{n}\left[\frac{\tau_{(\vec{k})} e^{e \hbar}}{m_{n}^{*}} * \frac{\partial f_{n}}{\partial \varepsilon} *(\vec{k} \cdot \varepsilon)\right] d \tau_{k}$

Pero, por la ecuación semiclásica de movimiento sabemos que:

$$
\begin{gathered}
p=\hbar * \vec{k} \\
m^{*} v=\hbar * \vec{k} \\
v=\frac{\hbar * \vec{k}}{m^{*}}
\end{gathered}
$$

(22) en (21):

$J_{n}=-\frac{e^{2} \hbar^{2}}{4 \pi^{3}\left[m_{n}^{*}\right]^{2}} * \int_{\left(V_{B}\right)} \tau_{e_{(\vec{k})}} * \frac{\partial f_{n}}{\partial \varepsilon} *(\vec{k} \cdot \vec{\varepsilon}) \vec{k} d \tau_{k}$

Donde:

$$
\tau_{e_{(\vec{k})}} \equiv \text { Tiempo de relajación para los electrones }
$$




\section{Conclusiones}

En la sección 2 se hace referencia a la clase de semiconductor sobre el cual tratará el estudio; es decir, el semiconductor debe ser homogéneo - no degenerado. Adicionalmente se consideran superficies iso-energéticas, las cuales para una partícula libre, tienen forma esférica.

En esta sección, también se hace referencia a la función de distribución de desequilibrio, la cual depende de la energía de la partícula, de sus coordenadas y del tiempo y se introduce la definición elemental de densidad de corriente.

La sección 3 empieza definiendo la función de distribución de desequilibrio y considera que la función de distribución de equilibrio es igual a cero por que se encuentra en equilibrio termodinámico por tanto se considera que, en este punto, la densidad de corriente equivale a cero.

La sección 4 está dedicada a deducir la ecuación cinética en la aproximación $\tau$, la cual proviene de la función de distribución de equilibrio más una perturbació... $\tau^{\prime}$

Finalmente la sección 5, se inicia con la deducción de la ecuación de la densidad de corriente para los electrones en función del tiempo de relajación, introduciendo en esta sección una definición muy escueta de masa efectiva del electrón y además se introduce la ecuación semi-clásica del momento lineal.

\section{Referencias}

[1] Bemsky, G. (1980). Semiconductores. Caracas: OEA.

[2] Espejo - López. O. L. (2004) Corriente eléctrica de portadores calientes en semiconductores intrínsecos : deseuilibrio en concentración . Revista Mexicana de física, p. 620.

[3] Shalímova. (1975). Física de los semiconductores. Moscú: Mir.

[4] Davidov, A. (1981). Teoría del sólido. Moscú: Mir.

[5] Goldstein, H. (1979). Mecánica clásica. Madrid: Addison - Wesley.

[6] Mermin (1976). Conductividad eléctrica de un metal. Física del Estado (págs. 7 - 11) New York: Hdt, Rinehart and Winston.

[7] Selby, S. (1965). Standard mathematical tables. Cleveland: The Chemical rubber Co.

[8] Pankove. (1971). Optical processes in semiconductors. New York: Dover Publications, Inc.

[9] Athmane Tadjine, 1 Y. M. (30 junio 2016) APS Physics Obtenido de http: // journals.aps.org/prb/abstract/10.1103/PhysRvB.95.235437 